\title{
Effect of Dental Follicle Mesenchymal Stem Cells on Th1 and Th2 Derived Naive T Cells in Atopic Dermatitis Patients
}

\author{
Noushin Zibandeh ${ }^{1}$ (D), Deniz Genc ${ }^{1}$ (D), Zuleyha Ozgen² (D), Yazgul Duran ${ }^{1}$ (i), Nurhan Kasap ${ }^{1}$ (D), Kamil Goker ${ }^{3}$ (D), Safa Baris ${ }^{1}$ (D), \\ Tulin Ergun² (iD), Tunc Akkoc ${ }^{1}$ (D) \\ ${ }^{1}$ Marmara University, School of Medicine, Division of Pediatric Allergy and Immunology, Istanbul, Turkey \\ ${ }_{2}^{2}$ Marmara University, School of Medicine, Department of Dermatology, Istanbul, Turkey \\ ${ }^{3}$ Marmara University, Faculty of Dentistry, Istanbul, Turkey
}

Correspondence Author: Tunc Akkoc

E-mail: tuncakkoc@gmail.com

Received: $11.04 .2018 \quad$ Accepted: 03.05.2018

\section{ABSTRACT}

Objective: The purpose of our study is to investigate the immunomodulatory effects of Dental Follicle Mesenchymal Stem Cells (DF-MSCs) on lymphocytes isolated from peripheral blood of Atopic Dermatitis (AD) patients, a Th2 disease and psoriasis, a Th1 / Th17 disease and compare them with healthy individuals in vitro.

Methods: Patients with the AD $(n=9)$ and psoriasis $(n=6)$ who are followed up in Marmara University Pediatric Allergy and Immunology and Dermatology outpatient clinics and healthy subjects $(n=6)$ were included. Peripheral Blood Mononuclear Cells (PBMCs) were isolated from $20 \mathrm{ml}$ of venous blood of all participants. Cells were cultured for 72 hours in the absence and presence of DF-MSCs with anti-CD3/anti-CD28 stimulation or without stimulation. At the end of this period, $\mathrm{CD} 4+$ and CD8+ T lymphocyte proliferation and cytokine levels from the culture supernatants were analyzed by flow cytometry.

Results: In the presence of DF-MSCs, proliferation ratio was suppressed in both CD4+ and CD8+ cells in AD and psoriasis patients ( $<<0,05)$. IFN- $\gamma$ levels significantly increased in AD patients in the presence of DF-MSCs $(p<0,05)$ whereas decreased significantly in psoriasis patients in the presence of DF-MSCs $(p<0,05)$. IL-4 levels significantly decreased in AD patients in the presence of DF-MSCs $(p<0,05)$ but remained unchanged in psoriasis patients $(p>0,05)$. IL-10 increased significantly in both groups in the presence of DF-MSCs $(p<0,05)$.

Conclusion: Our results support immunoregulatory effects of DF-MSCs on both AD and psoriasis which are Th2 and Th1 / Th17 dominant diseases respectively. Our evidence-based results demonstrated that DF-MSCs could have a beneficial therapeutic implication for inflammatory skin diseases. Keywords: Mesenchymal Stem Cells, Immunoregulation, Soluble factors, Atopic Dermatitis, Psoriasis

\section{INTRODUCTION}

Atopic Dermititis (AD) is a chronic inflammatory skin disease in which immune responses are mediated by Th2 (T helper 2) cells (1-3). It is one of the most common skin disorders with an estimated prevalence of up to $20 \%$ of children and $3 \%$ of adults $(4,5)$. It is characterized by xerosis, eczematous lesions, and severe pruritus $(6,7)$. There is a complex interplay between genetic, environmental and immunological factors in the pathogenesis of $A D(8)$. Psoriasis is, in contrast, a skin disease mediated mainly by Th1 and Th17 cells that produce IFN-y and IL-17 (9-11).

Several first-line treatments are available for antiinflammatory response to reduce the clinical symptoms of the AD and Psoriasis. Topical corticosteroids and systemic immunosuppressants and cytokine antagonis biologic therapies are used for treatment. However, none of these agents can provide a cure and have multiple side effects (1215). Accordingly there is an unmet need for more effective and safe therapeutic options in AD management. Mesenchymal Stem Cells (MSCs) can be a promising candidate because they can regulate multiple factors simultaneously in response to the inflammatory conditions $(16,17)$.
MSCs are the non-haematopoietic, multipotent stem cells with the capacity to differentiate into mesodermal lineage such as osteocytes, adipocytes and chondrocytes. MSCs express cell surface markers like cluster of differentiation CD29, CD44, CD73, CD90, CD105 and lack the expression of CD14, CD34, CD45 and HLA (Human Leucocyte Antigen)-DR (18). MSCs regulate the functions of various immune cells, including $T$ cells, B cells, natural killer cells, monocyte/ macrophages, dendritic cells, and neutrophils. T lymphocytes are the central mediators of many autoimmune and inflammatory diseases as well as of transplant rejection and graft-versus-host disease (19). Dental tissues provide a readily accessible source of MSCs (20). Among the dental origin MSCs, Dental follicle MSCs (DF-MSCs) show potent immunomodulatory properties which make them attractive approach for suppression of inflammatory conditions (21). Immunomodulation by MSCs is mediated by both direct cell-cell contact and release of soluble factors such as prostaglandin E2 (PGE2), indoleamine 2,3 dioxygenase (IDO), Transforming Growth Factor $\beta$ (TGF- $\beta$ ), released in response to stimulation by inflammatory cytokines (22-24).

Several recent studies have investigated the effects of Umbilical Cord-MSCs ve Bone Marrow-MSCs on antibody 
secreted B cell and mast cell degranulation in AD patients. Current data supports different and robust modulatory effects of MSCs, particularly on T cell response (25). Furthermore, the preventive or therapeutic potency and the mechanisms of action can be altered by changing the sources of MSCs.

The purpose of our study is to investigate the regulatory potential of DF-MSCs on $\mathrm{CD}^{+} \mathrm{T}$ helper (Th) and $\mathrm{CD}^{+} \mathrm{T}$ cytotoxic (TC) cell proliferation, inflammatory and antiinflammatory cytokine levels in AD patients and compare them wih psoriatics and healthy subjects.

\section{METHODS}

\subsection{Study Subjects}

Nine patients with an AD (mean age, 15,6 $\pm 1,62$ years) who fulfilled the criteria of Hanifin and Rajka (R) are included in the study. All patients showed positive cutaneous reaction to house dust mite. Patients showed total IgE of $>400 \mathrm{IU} / \mathrm{mL}$ (mean, 2650 $\pm 506,99 \mathrm{IU} / \mathrm{mL}$ ). None of the patients had any systemic immunosuppressive treatment for at least 2 months before testing and don't have any other chronic disease. Six Psoriasis patients (mean age, 22,33 $\pm 4,68$ years) are included. Their mean serum IgE level was 19,5 $\pm 6,09 \mathrm{IU} / \mathrm{mL}$. None of the patients had any systemic immunosuppressive treatment for at least 2 months before testing. Six healthy controls (mean age, $24,5 \pm 3,03$ years) with no history of atopy are included in the study as controls. Their mean serum IgE level was $17 \pm 1,2 \mathrm{IU} / \mathrm{mL}$. (Table 1 ).

This study is approved by Ethics Committee of the Marmara University Medical Faculty in Istanbul, Turkey (Protocol No: 09.2016.196/70737436-050.06.04). Written informed consent was obtained from all patients.

Table 1. Demographic data and clinical characteristics of the Patients. AD; Atopic Dermatitis patients (new diagnosed and non-treated), Ps; Psoriasis patients (new diagnosed and non-treated), C; Control group including HC. F: Female; M: Male; DF: Dermatophagoides farinae;DP: Dermatophagoides pteronyssinus, N: Negative.

\begin{tabular}{|c|c|c|c|c|c|}
\hline Patients & Age & Gender & $\begin{array}{l}\text { Total IgE } \\
\mathrm{U} / \mathrm{ml}\end{array}$ & SPT(mm) & Topical Therapy \\
\hline AD1 & 18 & $M$ & 2423 & DF: $5 * 6$, DP $: 6^{*} 6$ & N \\
\hline AD2 & 14 & M & 2748 & DF: $6 * 5, \mathrm{DP}: 6 * 6$ & $\mathrm{~N}$ \\
\hline AD3 & 15 & $\mathrm{~F}$ & 2105 & DF: $3 * 3, \mathrm{DP}: 4 * 3$ & $\mathrm{~N}$ \\
\hline AD4 & 14 & $M$ & 2867 & DF: $5 * 5$, DP: $4 * 4$ & Y \\
\hline AD5 & 14 & $M$ & 2374 & DF: $6 * 6$, DP: $5 * 6$ & N \\
\hline AD6 & 13 & $\mathrm{~F}$ & 3715 & DF: $4 * 5, D P: 5 * 5$ & Y \\
\hline AD7 & 22 & M & 2604 & DF: $6 * 6, D P: 5 * 6$ & $N$ \\
\hline AD8 & 17 & M & 2031 & DF: $4 * 5$, DP: $6 * 6$ & $N$ \\
\hline AD9 & 18 & M & 2987 & DF: $5 * 5, D P: 5 * 4$ & $N$ \\
\hline Ps1 & 28 & $\mathrm{~F}$ & 28 & $\mathrm{~N}$ & $N$ \\
\hline Ps2 & 22 & $\mathrm{~F}$ & 15 & $N$ & $N$ \\
\hline Ps3 & 17 & M & 11 & $N$ & $N$ \\
\hline Ps4 & 20 & M & 24 & $\mathrm{~N}$ & $N$ \\
\hline Ps5 & 19 & M & 20 & $N$ & $N$ \\
\hline Ps6 & 28 & $\mathrm{~F}$ & 19 & $\mathrm{~N}$ & $N$ \\
\hline $\mathrm{HC1}$ & 22 & $\mathrm{~F}$ & 25 & $\mathrm{~N}$ & N \\
\hline $\mathrm{HC2}$ & 23 & $\mathrm{~F}$ & 15 & $\mathrm{~N}$ & $\mathrm{~N}$ \\
\hline $\mathrm{HC} 3$ & 25 & $M$ & 23 & $\mathrm{~N}$ & N \\
\hline $\mathrm{HC} 4$ & 20 & $M$ & 10 & $\mathrm{~N}$ & $\mathrm{~N}$ \\
\hline HC5 & 27 & M & 28 & $N$ & N \\
\hline HC6 & 30 & $\mathrm{~F}$ & 17 & $\mathrm{~N}$ & $\mathrm{~N}$ \\
\hline
\end{tabular}

\subsection{Isolation and culture of DF-MSCS}

Dental follicle tissues were collected from 4 healthy volunteers aged between 19-25 years who have no abscess or inflammatory diseases. Third molar teeth were surgically removed before its eruption, and the dental follicle was extracted under sterile conditions. Dental follicle was cut into approximately $0,5 \mathrm{~mm}$ of diameter pieces and digested with $3 \mathrm{mg} / \mathrm{mL}$ collagenase type I (Sigma) in PBS (Invitrogen, USA) containing penicillin-streptomycin (Gibco, USA) for 45 $\min$ at $37^{\circ} \mathrm{C}$. The obtained cell suspensions were washed for two times and resuspended in DMEM containing \%10 Fetal Bovine Serum (FBS) and 1\% P/S (referred as complete DMEM) and cultured in $25 \mathrm{~cm}^{2}$ culture flasks. Non-adherent cells were removed by changing the cultivation medium. 


\subsection{Characterization and Determination of Multipotency of DF-MSCS}

DF-MSCs were characterized and differentiated into osteogenic, chondrogenic and adipogenic lineages in the third passage, in order to determine the multipotency of these cells. To evaluate the expression of surface markers, DFMSCs were trypsinized with 0,25\% trypsin EDTA and washed in phosphate-buffered saline (PBS; Gibco, Gaithersburg, $M D)$. After 15 minutes of incubation period with FITC or PEconjugated mouse anti-human antibodies specific to CD34, CD45, CD14, CD29, CD44, CD73, CD90, CD105, or HLA-DR, cells were analyzed via flow cytometer (FACSCalibur; BD Biosciences, San Jose, CA) with CellQuest software. The mouse IgG served as isotype control.

DF-MSCs were stimulated with StemPro ${ }^{\circledR}$ Osteogenesis Differentiation (Gibco, Grand Island, NY), StemPro ${ }^{\circledR}$ Adipogenesis Differentiation Kit (Gibco) and StemPro ${ }^{\circledR}$ Chondrogenesis Differentiation Kit (Gibco) according to manufacturer's protocol to differentiate into osteocytes, adipocytes, and chondrocytes. Briefly, cells $\left(1 \times 10^{5} /\right.$ well $)$ were seeded in 6 -well plates and 3 days after seeding the cells were replaced with differentiation mediums. The cells were grown for 3 weeks, with medium replacement twice a week. Osteogenesis was detected by staining with Alizarin Red to determine extracellular calcium deposits. Adipogenesis was determined by Oil Red $O$ to stain oil droplets produced by adipocytes. Chondrogenesis was assessed by Alcian Blue staining to determine extracellular proteoglycans. All Images of stained cells were captured by using a light microscope.

\subsection{Isolation of PBMCs from Whole Blood Samples}

Twenty milliliters of peripheral blood was collected in heparinized tubes prior to isolation procedure. Peripheral blood mononuclear cells (PBMCs) were isolated by density gradient centrifugation for $20 \mathrm{~min}$ at $2000 \mathrm{rpm}$. PBMCs were resuspended in complete culture medium (RPMI-1640, supplemented with $1 \% \mathrm{P} / \mathrm{S}, 10 \% \mathrm{FBS}$, all from Invitrogen) after cell counting by hemocytometer and adjusted to a final concentration of $5 \times 10^{5}$ cells/well in 48 well plates (26).

\subsection{Co-culture of DF-MSCs with PBMC}

In co-cultures, DF-MSCs $\left(5 \times 10^{4 /}\right.$ well in a 48 -well plate) were plated $48 \mathrm{~h}$ before the addition of $5 \times 10^{5}$ of PBMCs (in the ratio of $1: 10$ ) with complete RPMI-1640 medium (RPMI 1640 containing $\% 10 \mathrm{FBS}$ and $1 \% \mathrm{P} / \mathrm{S}$ ) and were co-cultured for 3 days. T lymphocytes were stimulated using 0,5 $\mu \mathrm{g} /$ $\mathrm{ml}$ anti-CD3 (eBioscience, San Diego, CA) and $0,5 \mu \mathrm{g} / \mathrm{ml}$ anti-CD28 (eBioscience, San Diego, CA) antibodies (21).

\subsection{Lymphocyte Proliferation Assay}

In order to determine the effect of DF-MSCs on proliferative response of $\mathrm{CD}^{+}$or $\mathrm{CD}^{+} \mathrm{T}$ lymphocytes, $\mathrm{PBMC}$ was labeled with Carboxyfluorescein succinimidyl ester (CFSE) prior to culturing. Briefly, each $1-2 \times 10^{6}$ PBMC was diluted in $1 \mathrm{~mL}$ of
PBS with $18 \mathrm{mM}$ of CFSE and incubated for 6 minutes at $4^{\circ} \mathrm{C}$ in dark conditions. After incubation period cells were washed twice with culture medium (completeRPMI), supernatant was discharded and remaining cell pellet was resuspended in culture medium before culturing. Cells were analyzed for CFSE (FITC) signaling via flow cytometry after 3 days of culture period (27).

\subsection{Analysis of Cytokine Expression Profiles}

Supernatant from cultures was collected and stored at 80 ㅇ C until assayed. Samples were measured and analyzed for IFN- $\gamma$, IL-4 and IL-10 cytokine levels by Cytokine Bead Array (CBA) kit (BD Biosciences, USA) according to the manufacturer's protocol. Briefly, all the CBA kit contents and samples should be at room temperature at least 15 minutes. fifty microliters of culture supernatants, fifty microliters of capture beads and fifty microliters of detection reagent were added and incubated for 3 hours. After incubation, samples were washed for two times with cold PBS. Samples were acquired in a FACS Calibur flow cytometer (BD Biosciences) and analyzed using the FCAP Array v1.0.1 software (Soft Flow Inc.). Results were expressed as picograms per milliliter.

\subsection{Down Regulation of IDO, PGE-2 and TGF-B}

To explore the crucial factors responsible for the suppressive effect of DF-MSCs on T cytokine secretion, we inhibited the synthesis or the action of crucial factors using selective inhibitors. The crucial molecules or signals such as transforming growth factor (TGF)- $\beta 1$, indoleamine-2, 3-dioxygenase-1 (IDO-1), and cyclooxygenase-2 (COX-2) were down-regulated with anti-TGF- $\beta 1(0.5 \mu \mathrm{g} / \mathrm{ml})$ neutralizing antibody, 1-methyl tryptophan (MDT, 0,1 Mm,) 1-Methyl-Dtryptophan (1-MDT) and SC-58125 $(50 \mu \mathrm{M}$,$) respectively. After$ culture period, supernatants were analyzed for the cytokines levels via flow cytometry.

\subsection{Statistical analysis}

The statistical analysis was achieved by using GraphPad Prism 5 (GraphPad Software, La Jolla, CA, USA). One way analysis of variance (ANOVA) with Tukey's multiple comparisons was used for multi-group comparisons, and a two-tailed unpaired Student's t-test was used for comparisons between two groups, and a p-value of $<0.05$ was considered statistically significant.

\section{RESULTS}

\subsection{Isolation, Characterization and Differentiation of DF- MSCs}

The MSCs were isolated from dental follicle tissues. Their proliferation gradually formed small colonies in 3 days. The MSCs reached $70 \%$ confluency in the primary culture 7 days after plating for the first passage. Most of the MSCs exhibited fibroblast-like morphology at the P3 passage (Figure 1A).

The MSCs were analyzed via flow cytometry. These cells exhibited positive staining for CD29, CD90, CD 146, CD73 and 
CD106 but were negative for CD34, CD45, CD14, CD28 and CD25 (Figure 1B).

The MSCs differentiated into osteocytes, adipocytes, and chondrocytes. First, the osteogenic differentiation capability was investigated in vitro during a twenty-eightday culture period in osteogenic induction medium. The MSCs were stained with Alizarin red and the cells formed calcified bone nodule structures. Next, in vitro adipogenic differentiation capability was assessed by culturing the cells in adipogenic induction medium and staining with Oil Red O. Intracellular lipid droplets was observed in these cells. Chondrogenic differentiation capability was investigated in vitro following fourteen-day culture period in chondrogenic induction medium and cell differentiation into chondrocytes was confirmed with Alcian blue staining which exhibited intracellular proteoglycans in those cells (Figure 1C).
A)
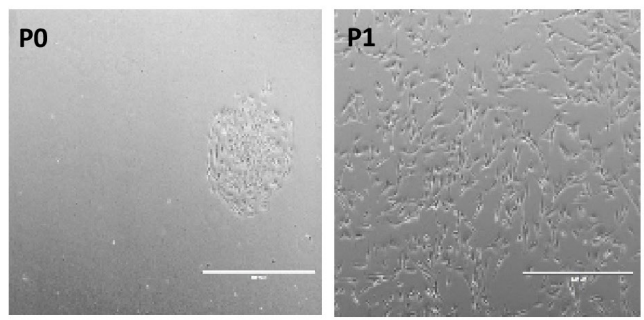
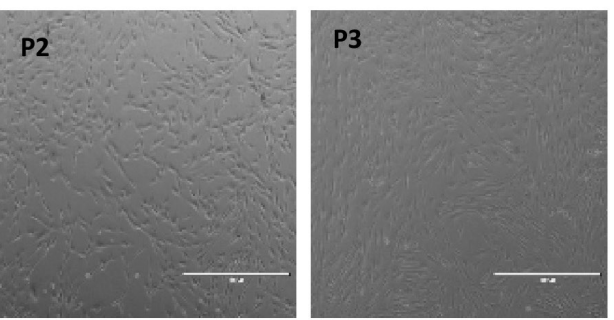

B)
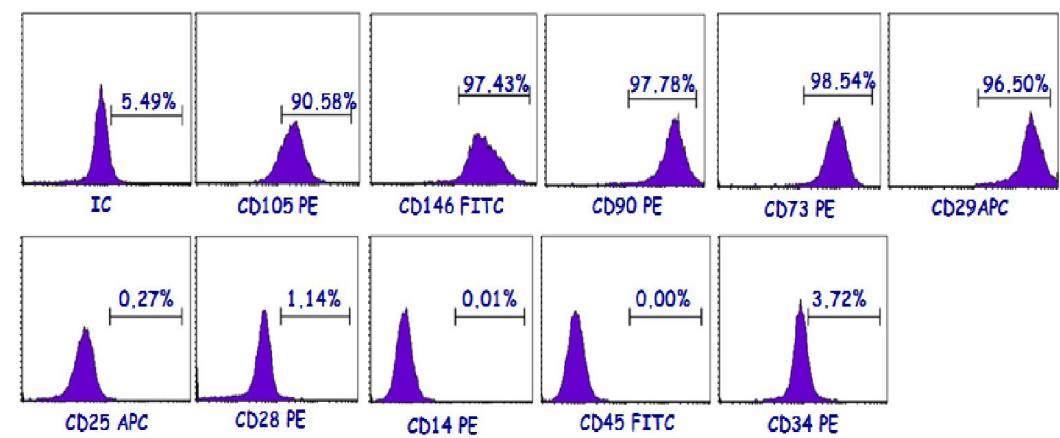

C)

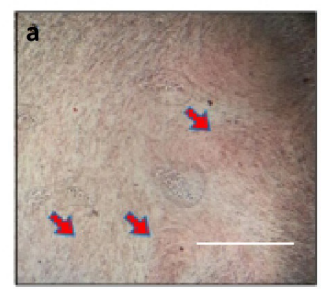

Osteogenic

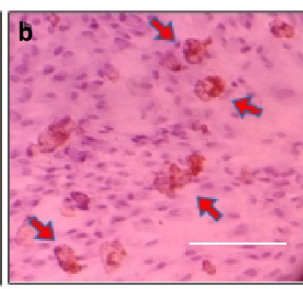

Adipogenic

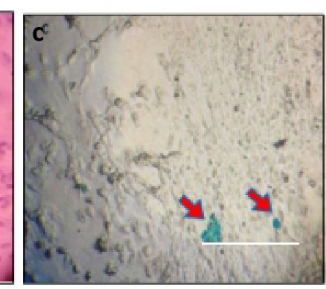

Chondrogenic

Figure 1. Morphological appearance, characterization, and differentiation of DFSCs. A). Morphology of DFSCS in $P O, P 1, P 2$, and $P 3$ (magnification =10x). B). Representative flow cytometry analysis of positive surface markers CD105, CD146, CD90, CD73,CD29 and negative surface marker CD25, CD28, CD14, CD45, CD34 for DFSCs at the third passages. C). (a)Alizarin red staining of osteogenic induced DFSCs (b) Oil Red staining of adipogenic induced DFSCS (c) Alcian blue staining of chondrogenic induced DFSCs, scale bar $=1000 \mu \mathrm{m}$.

\subsection{DF-MSCs Decreased Proliferative Response of Lymphocytes in AD Patients}

We investigated the immunomodulatory effect of DF-MSCs on $\mathrm{CD}^{+} \mathrm{Th}$ and $\mathrm{CD} 8^{+} \mathrm{T}$ cell phenotypes in $\mathrm{AD}$ and Psoriasis Patients by CFSE cell labeling. According to our results, $\mathrm{CD} 4^{+}$Th proliferation capacity was significantly higher in $\mathrm{AD}$ and Psoriasis compared to healthy controls $(p<0.01, p<0.05$, respectively). $C D 4^{+} T$ cells proliferation was decreased in the presence of DF-MSCs in both $A D$ and Psoriasis group, but it was not shown a significant difference in healthy controls ( $p<0.01, p<0.05, p>0.05$, respectively). CD8 ${ }^{+} T c$ proliferation capacity tended to increase in $A D$ and Psoriasis compared to healthy controls but it is not statistically significant $(p>0.05$, $p>0.05$, respectively).

$\mathrm{CD}^{+} \mathrm{Th}$ cells proliferation was decreased in the presence of DF-MSCs in both AD and Psoriasis group, but it was not shown a significant difference in healthy controls $(p<0.001$, $p<0.05, p>0.05$, respectively) (Figure2). 


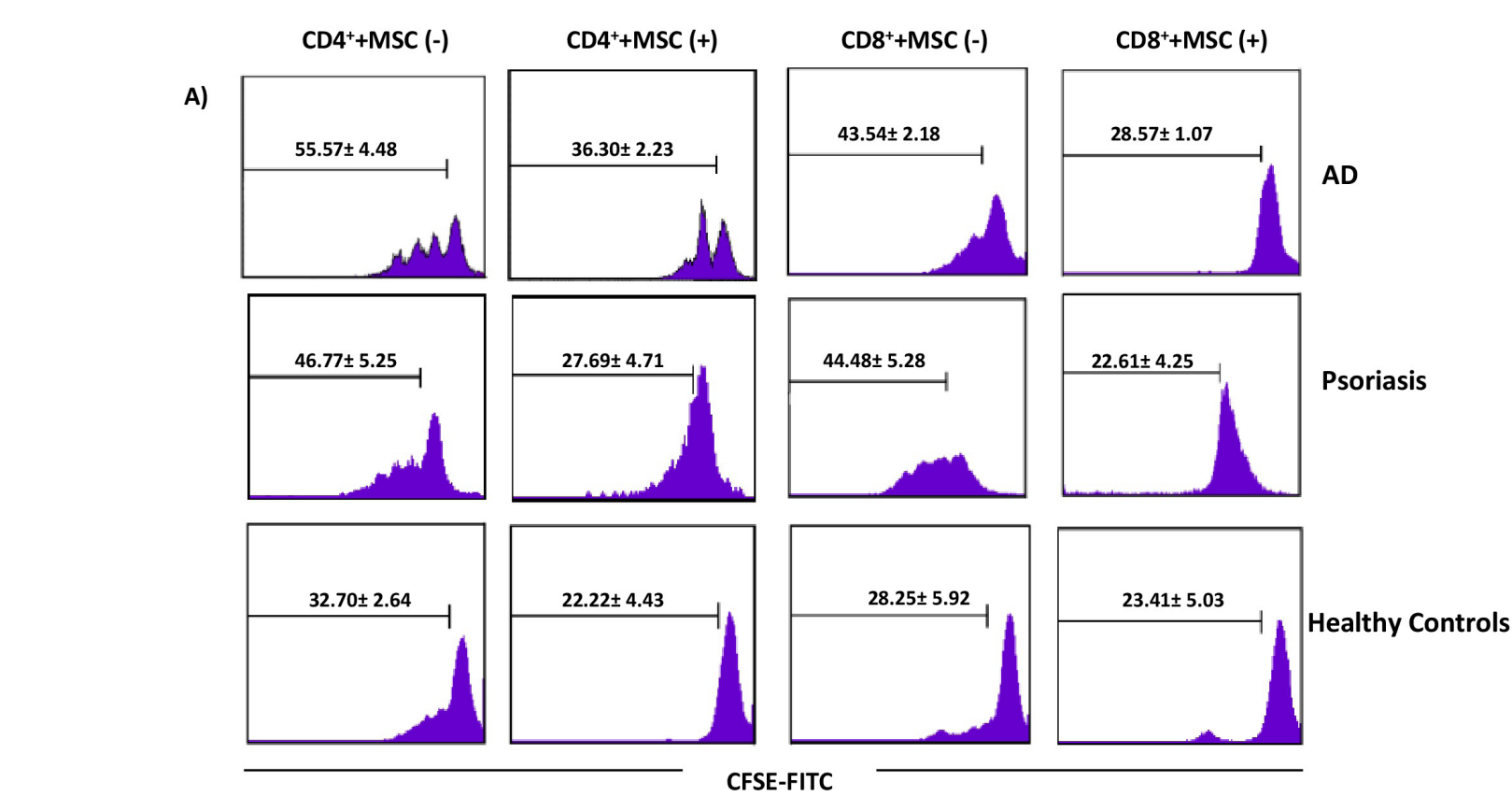

B)

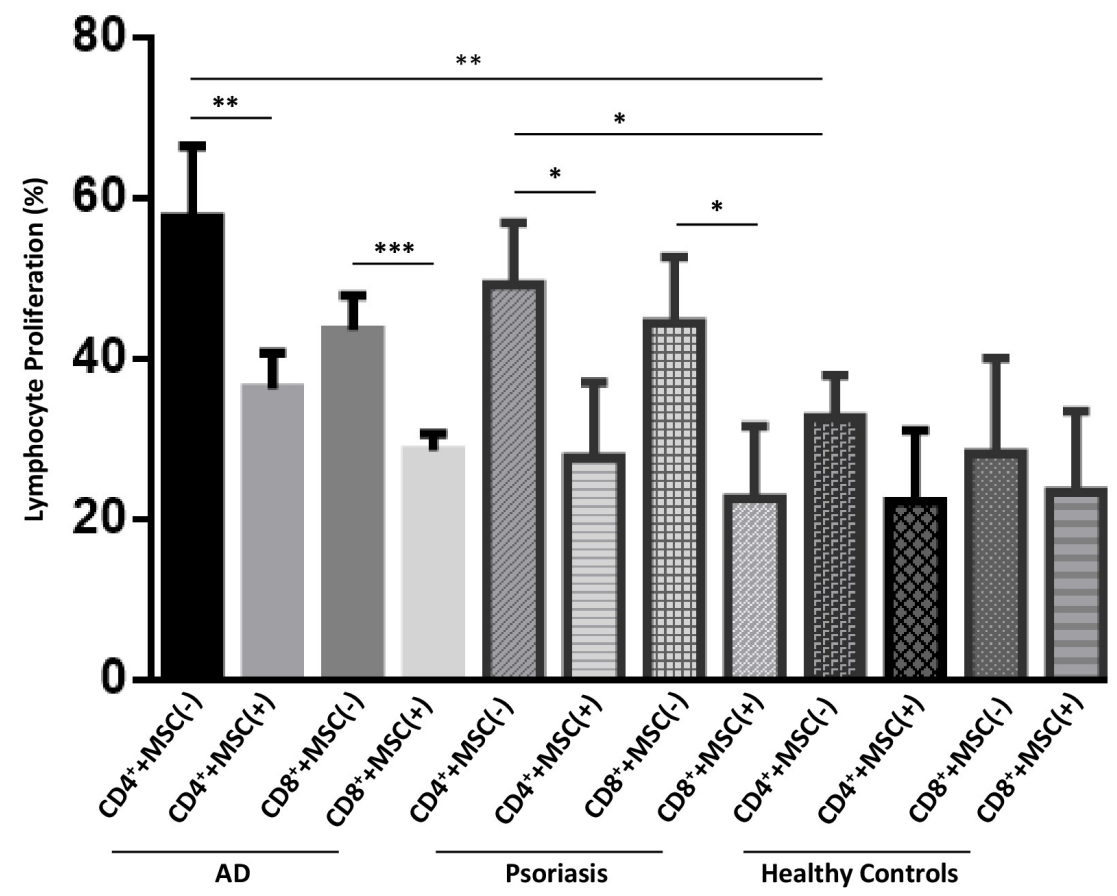

Figure 2. Inhibitory effect of DF-MSCS on the proliferation of lymphocytes as detected by CFSE. A) Inhibitory effect of DF-MSCS on the proliferation of lymphocytes displayed by flow cytometry. B) Inhibitory effect of DF-MSCS on the proliferation of lymphocytes displayed statistically. ${ }^{*} P<0.05$. Results are shown as mean $\pm S D$.

\subsection{DF-MSCs Regulate the Inflammatory and Anti- Inflammatory Cytokine Production in CDmix-Stimulated PBMCs in AD Patients.}

We investigated the immunomodulatory effect of DFMSCs on Th cell phenotypes by evaluating IFN- $\gamma$ levels for Th1 and IL-4 levels for Th2 cells, and IL-10 levels as an antiinflammatory cytokine mainly produced by $\mathrm{T}$ regulatory cells. Culture supernatants were collected on 3 day of culture period and analyzed with CBA kit via flow cytometry. IL-4 levels were significantly high and IFN- $\gamma$ levels were low in AD patients' PBMC cultures compared to healthy subjects and PS patients $(p<0,05)$. DF-MSCs significantly decreased IL-4 levels and increased IFN- $\gamma$ levels in co-cultures of $A D$ patients $(p<0,05)$, while decreased IFN- $\gamma$ levels in co-cultures of PS patients $(p<0,05)$. There was no significant difference between DF-MSC (-) and DF-MSC (+) groups in healthy controls $(p>0,05)$. IL-10 levels were significantly increased with DF-MSCs in AD patients and PS patients $(p<0,005$ and $p<0,01$, respectively), and tend to increase in healthy controls but it was not significant. There is not significant difference in IFN- $\gamma$, IL-4 an IL-10 cytokine levels between PGE-2, TGF- $\beta$ and IDO blockade culture supernatant in AD, Psoriasis and healthy controls (Figure 3). 

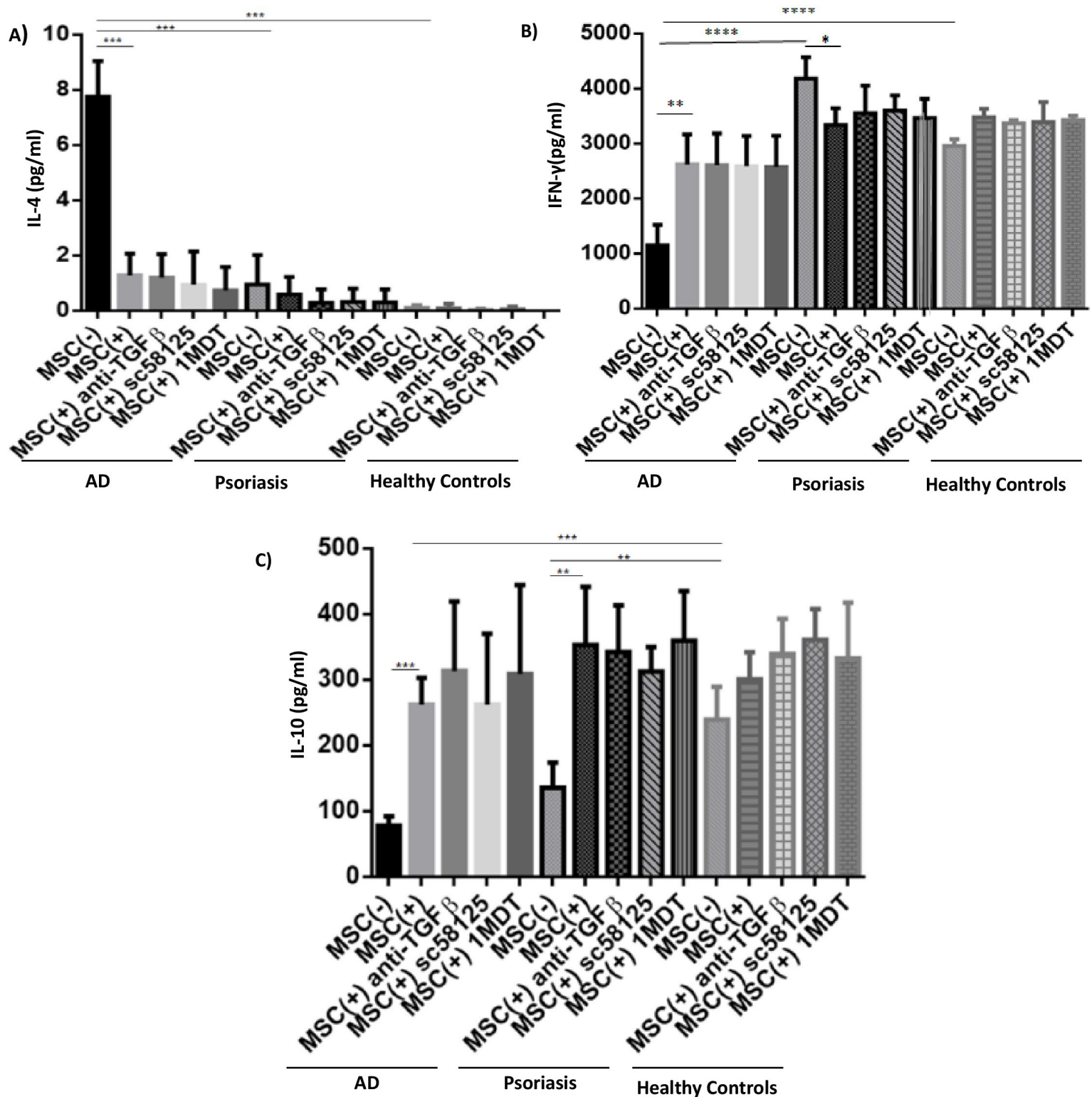

Figure 3. DF-MSCs-modulated cytokine levels in the supernatants of CDmix-stimulated PBMCs. PBMCS (5 $\times 105$ cells/well) collected from patients with the $A D$, Psoriasis, and $\mathrm{HC}$ were cultured in a 48-well plate following CDmix $(0.5 \mu \mathrm{g} / \mathrm{ml}, 0.5 \mu \mathrm{g} / \mathrm{ml})$ stimulation in the presence or absence of DF-MSCS $(5 \times 104 /$ well) for 3 days. The levels of IFN- $\gamma, I L-4$, and IL-10 in the supernatants of CDmix-stimulated PBMCS were determined using flow cytometry. DF-MSCs significantly decreased IL-4(A) ( $p \leq 0.001)$ but significantly increase IFN- $\gamma(B)$ and IL-10(E) levels ( $p \leq 0.01, p \leq 0.001$, respectively) in AD patients. DF-MSCs significantly decreased IFN- $\gamma(B)(p \leq 0.05)$ but significantly increase IL-10 levels (E) $(p \leq 0.01)$ in Psoriasis patients. IL-4 was not shown a significant difference in presence of DF-MSCs in Psoriasis patients. ${ }^{*} P<0.05$. Results are shown as mean $\pm S D$.

\section{DISCUSSION}

$A D$ is a common chronic skin disease. The currently available therapeutics are limited, and AD management becomes challenging in most cases. To develop better therapeutics for the treatment of $A D$, many studies have focused on the pathogenesis of AD. Historically, AD was considered a Th2 cell-mediated disease. Experimental and clinical evidence suggests that Th2 cells and their related cytokines like IL-4 and chemokines are critical for the pathogenesis of AD (13). Therefore, many researchers have targeted Th2 cells or its signature cytokine IL-4 to treat AD. Indeed, human anti-IL-4 antibody (Dupilumap) effectively treats AD (28). Recently, MSCs have been shown to have immunosuppressive effects Furthermore, it has been proposed that MSCs could inhibit Th2 cell differentiation by reducing IL-4 cytokine level (29). These discoveries of immunosuppressive function and the ability to inhibit lymphocytes have driven scientists to test the possibility that MSCs can be used for the treatment of allergic inflammation diseases because Th2 cells are significant targets of allergic inflammation diseases. In this study, we demonstrated that DF-MSCs could inhibit T cells proliferation capacity and reduce IL-4 cytokine levels in AD patients. 
Immunomodulatory effects of MSCs make them new candidates as a cellular therapy for the prevention and treatment of various inflammatory diseases (30). Mesenchymal stem cells can be isolated from many different tissues which exhibit remarkable tissue regenerative and immunosuppressive properties (31). Among these cells, dental tissue MSCs represent a source that is easily accessible and have the potential to differentiate into other tissue cell lines (32). Yıldırım and Colleagues show that DF-MSCs reduced inflammatory response compared to other dental sources (21). We investigate DF-MSCs immunomodulatory effect in inflammatory skin diseases for the first time.

MSCs suppress the response of T cells to stimulation factors. In a previous study, Bone marrow-derived stem cells (BMMSCs) suppress T cell proliferation in OVA albumin induced atopic dermatitis mouse model (17). Although Th2-type CD4 ${ }^{+}$ Th cells appear to be significant in $A D$ pathogenesis, $C D 8^{+}$ Tc cells represent the dominant effector cell responses in the development of the allergic skin inflammation (33). To examine the $C D 4^{+} T h$ and $C D 8^{+} T c$ cell in $A D$ pathogenesis, we investigated the proliferative response of both $T$ cell types in the presence of DF-MSCs. According to our results, DF-MSCs decrease $C D 4^{+} T h$ and $C D 8^{+}$Tc cell response in $A D$ patients. This suppression was seen in the $\mathrm{CD} 4^{+} \mathrm{Th}$ and $\mathrm{CD} 8^{+} \mathrm{Tc}$ cells of Th1/Th17 -type psoriasis patients. For the first time, we investigate the role of DF-MSCs on the response of $\mathrm{CD} 4^{+} \mathrm{Th}$ and $\mathrm{CD} 8^{+} \mathrm{Tc}$ in two different inflammatory skin disease.

$\mathrm{Na}$ and colleagues showed that BM-MSCs suppress both IL-4 and IFN $-\gamma$ through decreasing T-bet and GATA-3 expression (17). According to Fu and colleagues, BM-MSCs increased IFN $-\gamma$ cytokine levels in allergic rhinitis with Th2-skewed eosinophilic inflammation but reduce IL-4 levels (25). After of Lymphocytes with DF-MSCs, the supernatants were collected, and cytokines analysis were performed. In our study, DF-MSCs suppressed the expression of IL-4 whereas the expression of IFN $-\gamma$ and IL-10 was increased in AD patients. DFMSCs increased IFN $-\gamma$ levels in Th1/Th17 skewed Psoriasis patients, but IL-4 levels were not changed. According to our results, DF-MSCs provides immunomodulation according to the characteristics of lymphocyte in the culture.

\section{CONCLUSION}

These inhibitory actions might contribute to the therapeutic effects of DF-MSCs on both AD and Psoriasis patients. To our knowledge, these findings provide the new perspective that MSCs manipulation is a potential novel strategy for the treatment of inflammatory skin disease.

\section{REFERENCES}

[1] Drucker AM. Atopic dermatitis: burden of illness, quality of life, and associated complications. Allergy Asthma Proc 2017; 38: 3-8.

[2] Bieber T. Atopic dermatitis. Ann Dermatol 2010; 22: 125-137.
[3] Brunner PM, Guttman-Yassky E, Leung DY. The immunology of atopic dermatitis and its reversibility with broad-spectrum and targeted therapies. J Allergy Clin Immunol 2017; 139: 65-76.

[4] Nutten S. Atopic dermatitis: Global epidemiology and risk factors. Ann Nutr Metab 2015; 66: 8-16.

[5] Shaw TE, Currie GP, Koudelka CW, Simpson EL. Eczema prevalence in the United States: Data from the 2003 National Survey of Children's Health. J Invest Dermatol 2011; 131: 67-73.

[6] Leung DY, Nicklas RA, Li JT, Bernstein IL, Blessing- Moore J, Boguniewicz M, Chapman JA, Khan DA, Lang D, Lee RE, Portnoy JM, Schuller DE, Spector SL, Tilles SA. Disease management of atopic dermatitis: an updated practice parameter. Joint Task Force on Practice Parameters. Ann Allergy Asthma Immunol 2004; 93: 1-21.

[7] Bieber T. Atopic dermatitis. N Engl J Med 2008; 358:14831494.

[8] Brunner PM, Leung DYM, Guttman-Yassky E. Immunologic, microbial, and epithelial interactions in atopic dermatitis. Ann Allergy Asthma Immunol 2018; 120: 34-41.

[9] Lowes MA, Bowcock AM, Krueger JG. Pathogenesis and therapy of psoriasis. Nature 2007; 445: 66-73.

[10] Guttman-Yassky E, Lowes MA, Fuentes-Duculan J, Whynot J, Novitskaya I, Cardinale I, Haider A, Khatcherian A, Carucci JA, Bergman $R$, Krueger JG. Major differences in inflammatory dendritic cells and their products distinguish atopic dermatitis from psoriasis. J Allergy Clin Immunol 2007; 119: 1210-1217.

[11] Org PY, Leung DY. Immune dysregulation in atopic dermatitis. Curr Allergy Asthma Rep 2006; 6: 384-389.

[12] Khattri S, Shemer A, Rozenblit $M$, Dhingra N, Czarnowicki T, Finney R, Gilleaudeau P, Sullivan-Whalen $M$, Zheng $X, X u H$, Cardinale I, de Guzman Strong C, Gonzalez J, Suárez-Fariñas M, Krueger JG, Guttman-Yassky E. Cyclosporine in patients with atopic dermatitis modulates activated inflammatory pathways and reverses epidermal pathology. J Allergy Clin Immunol 2014; 133: 1626-1634.

[13] Gooderham M, Lynde CW, Papp K, Bourcier M, Guenther L, Gulliver W, Hong CH, Poulin Y, Sussman G, Vender R. Review of systemic treatment options for adult atopic dermatitis. J Cutan Med Surg 2016: 21:31-39.

[14] Diaz A, Guttman-Yassky E. Topical agents for the treatment of atopic dermatitis. Expert Rev Clin Immunol 2019; 15:369-382.

[15] Tintle S, Shemer A, Suárez-Fariñas $M$, Fujita $H$, Gilleaudeau $P$, Sullivan-Whalen $M$, Johnson-Huang $L$, Chiricozzi $A$, Cardinale I, Duan S, Bowcock A, Krueger JG, Guttman-Yassky E. Reversal of atopic dermatitis with narrow-band UVB phototherapy and biomarkers for therapeutic response. J Allergy Clin Immunol 2011; 128: 583-593.

[16] Kim HS, Yun JW, Shin TH, Lee SH, Lee BC, Yu KR, Seo Y, Lee S, Kang TW, Choi SW, Seo KW, Kang KS. Human umbilical cord blood mesenchymal stem cell-derived PGE2 and TGF-beta1 alleviate atopic dermatitis by reducing mast cell degranulation. Stem cells 2015; 33:1254-1266.

[17] Na K, Yoo HS, Zhang YX, Choi MS, Lee K, Yi TG, Song SU, Jeon MS. Bone marrow-derived clonal mesenchymal stem cells inhibit ovalbumin-induced atopic dermatitis. Cell Death Dis 2014; 5: e1345.

[18] Barry FP, Murphy JM. Mesenchymal stem cells: clinical applications and biological characterization. Int J Biochem Cell Biol 2004; 36: 568-584. 
[19] Duffy MM, Ritter T, Ceredig R, Griffin MD. Mesenchymal stem cell effects on T-cell effector pathways. Stem Cell Res Ther 2011; 2: 34.

[20] Saito M. T, Silverio K. G, Casati M. Z, SallumE. A, Nociti F. H. Jr Tooth-derived stem cells: Update and perspectives. World J Stem Cells 2015; 7: 399-407.

[21] Yildirim S, Zibandeh N, Genc D, Ozcan EM, Goker K, Akkoc T. The Comparison of the Immunologic Properties of Stem Cells Isolated from Human Exfoliated Deciduous Teeth, Dental Pulp, and Dental Follicles. Stem Cells Int 2016; 4682875: 105-106.

[22] Krampera $M$, Cosmi L, Angeli R, Pasini A, Liotta F, Andreini A, Santarlasci V, Mazzinghi B, Pizzolo G, Vinante F, Romagnani P, Maggi E, Romagnani S, Annunziato F. Role for interferon-gamma in the immunomodulatory activity of human bone marrow mesenchymal stem cells. Stem Cells 2006; 24: 386-398.

[23] Ryan JM, Barry F, Murphy JM, Mahon BP. Interferon-gamma does not break, but promotes the immunosuppressive capacity of adult human mesenchymal stem cells. Clin Exp Immunol 2007; 149: 353-363.

[24] DelaRosa O, Lombardo E, Beraza A, Mancheno-Corvo P, Ramirez C, Menta R, Rico L, Camarillo E, García L, Abad JL, Trigueros C, Delgado M, Büscher D. Requirement of IFN-gamma-mediated indoleamine 2,3-dioxygenase expression in the modulation of lymphocyte proliferation by human adipose-derived stem cells. Tissue Eng Part A 2009; 15: 2795-2806.

[25] Fu Q, Chow Y, Sun S, Zeng Q, Li H, Shi J, Sun YQ, Wen W, Tse $H F$, Lian $Q, X u G$. Mesenchymal stem cells derived from human induced pluripotent cells modulate $\mathrm{T}$ - cell phenotypes in allergic rhinitis. Allergy 2012; 67: 1215-1222.
[26] Fuss IJ, Kanof M, Smith P, Zola H. Isolation of whole mononuclear cells from peripheral blood and cord blood. Curr Protoc Immunol 2009; Chapter 7:Unit7.1.

[27] Quah B, Parish C. The Use of Carboxyfluorescein Diacetate Succinimidyl Ester (CFSE) to Monitor Lymphocyte Proliferation. J Vis Exp 2010; 4: 2259.

[28] Massimiliano D'Erme A, Romanelli M, Chiricozzi A. Spotlight on dupilumab in the treatment of atopic dermatitis: design, development, and potential place in therapy. Drug Des Devel Ther 2017; 11: 1473-1480.

[29] Iyer SS, Rojas M. Anti-inflammatory effects of mesenchymal stem cells: novel concept for future therapies. Expert Opin Biol Ther 2008; 8: 569-581.

[30] Lee YS, Sah SK, Lee JH, Seo KW, Kang KS, Kim TY. Human umbilical cord blood-derived mesenchymal stem cells ameliorate psoriasis-like skin inflammation in mice. Biochem Biophys Rep 2016; 9: 281-288.

[31] TW, Lim JH, Chung CJ, Kang TJ, Kim TY, Kim YS, Roh TS, Lew DH. Immune Tolerance of Human Dental Pulp-Derived Mesenchymal Stem Cells Mediated by CD4+CD25+FoxP3+ Regulatory T-Cells and Induced by TGF- $\beta 1$ and IL-10. Yonsei Med J 2017; 58: 1031-1039.

[32] Keyser KA, Beagles KE, Kiem HP. Comparison of mesenchymal stem cells from different tissues to suppress T-cell activation. Cell Transplant 2007; 16: 555-562.

[33] Hennino $A$, Vocanson $M$, Toussaint $Y$, Rodet $K$, Benetière $J$, Schmitt AM, Aries MF, Bérard F, Rozières A, Nicolas JF. Skininfiltrating CD8+ T cells initiate atopic dermatitis lesions. J Immunol 2007; 178: 5571-5577. 